\title{
On the Construction of a Theory of Everything Based on Buddhist Cosmological Model
}

\author{
Weicheng Cui ${ }^{1,2 *}$ and Linlin Kang ${ }^{3}$ \\ ${ }^{1}$ School of Engineering, Westlake University, China \\ ${ }^{2}$ Shanghai Engineering Research Center, Hadal Science and Technology, Shanghai Ocean University, China \\ ${ }^{3}$ Westlake Institute for Advanced Study, China
}

Submission: January 21, 2020; Published: February 14, 2020

*Corresponding author: Weicheng Cui, School of Engineering, Westlake University, Hangzhou, China

\begin{abstract}
Sciences originated from seeking the solutions to some fundamental questions about the universe and life. Earlier application of a holistic approach led to the first peak when some of the unfalsifiable theories such as Buddhism and Daoism were developed while introduction of a reductionist approach led to the born of Newtonian mechanics. After that sciences developed in a very fast speed but in a divergent way and many conflicts or paradoxes exist among different scientific theories. How to establish a logically consistent theory for everything is a target for some scientists and currently several versions of theory of everything (TOE) are available. In this paper, a new version of TOE is proposed, and the basic idea of this TOE is that the theoretical framework of Bertalanffy's general system theory is borrowed but its materialist philosophy is replaced by Buddhist philosophy. Based on six axioms, a general uncertainty principle is derived which can explain all the phenomena we observed. Of course, the validity of the TOE is subjected to more strict examination by other scientists.
\end{abstract}

Keywords: The Big-Bang Cosmological Model; The Buddhist Cosmological Model; Theory of Everything; General System Theory

\section{Introduction}

Where did we (our human beings) come from? Where should we go after dying? Who am I? When did the universe begin? What is the universe made of? How big is the universe? Will the universe end? Does the universe and life operate according to some laws or randomly? These and many other similar questions have been explored since very ancient times by many people including scientists, philosophers, religionists, and many theories have been proposed [1,2]. In ancient times there was no division of subject areas and people considered these problems together, so very often, a scientist was also a philosopher or a religionist such as Lao Zi (571-471 BC), Confucius (571-471 BC), Siddhārtha Gautama (563-483 BC), Aristotle (384-322 BC). This approach to view all the problems together is later defined as a holistic approach which means taking care of the system totally in all aspects. This earliest period from the origin of human beings to these greater philosophers can be regarded as the first peak time of scientific exploration. They applied a holistic approach to construct different theories to explain all the phenomena in the universe and Daoism, Buddhism and Greek Philosophy are typical examples of such theories. If one studies them carefully and makes a comparison one may find that Buddhism is the widest and deepest and it can cover all the theories of Daoism and Greek Philosophy [3]. Fundamentally speaking, there are two schools of philosophy, monism and dualism and within the monism, there are three types of ontology, matter, mind and God. Monism can be regarded as a special case of dualism. One of the significant characteristics of the holistic theories is that they pay more attention to correctness and sacrifice the accuracy, that is, they are qualitative rather than quantitative. Up to the author's knowledge, no scientific papers have been seen to reveal the outdated opinions in the Buddhism and Daoism. Later, we realized that correctness and accuracy are conflict in nature and there will always be a balance between these two for the given information. This can be called the accuracycorrectness balance principle: Accuracy and correctness are in conflict in the sense that the more accurate the representation of a statement, the higher the information content but the less likely it is to be correct [4].

Later, people introduced a reductionist approach to study the so-called objective problems and left the subjective life issues to religionists. The reductionist approach divides a mechanical 
system into several subsystems and studies the subsystems quantitatively by introducing many measurement methods. It was first divided into religion and science, the former studies the life while the later studies the universe. The main achievement of the former was the Christianity and this theory can explain quite a lot of phenomena after civilization but attributed all the unknowns to the concept of God. For the latter, an experimental approach was introduced to test the theory and Galileo Galilei was one of the big contributors. People who adopted the reductionist approach believe that explanation of a complex system can always be made in terms of their individual, constituent parts and their interactions. Since human beings live in the universe, there are some overlapping areas among scientists and religionists, there was a long war between scientists and religionists from $5^{\text {th }}$ century to $16^{\text {th }}$ century. Galileo observed the universe through the telescope and found it is different from what was told in Christian religion and through a list of great scientists' efforts, finally scientists won the war and Newtonian mechanics was born. This can be regarded as the second peak time of scientific exploration and people gradually accept that sciences are superior to the Christianity. Newtonian mechanics was so powerful and at that time, some scientists optimistically thought that Newtonian mechanics might be the final theory for the universe. On Friday, April 27, 1900, the British physicist Lord Kelvin gave a speech entitled "Nineteenth-Century Clouds over the Dynamical Theory of Heat and Light," which began: "The beauty and clearness of the dynamical theory, which asserts heat and light to be modes of motion, is at present obscured by two clouds" [5]. However, these two small clouds resulted in the separate developments of general relativity (GR) theory and quantum mechanics (QM). This can be regarded as the third peak time of science development. These two theories are based on two different philosophical foundations. While GR was still based on a causal-effect law, but
QM gave up that law and it was assumed that the nature of micro world is random. Heisenberg derived an uncertainty principle to emphasize this point $[6,7]$. Einstein was very unhappy with this interpretation of quantum phenomena [8]. However, from this development, we realize our limitation to know the external world, no matter macro or micro worlds. Due to this limitation, uncertainty will always exist for a complex system, whether these uncertainties can be reducible or not divided scientists into two schools. But the sudden jump from macro world to micro world is certainly hard to explain.

Later people applied the principles of Newtonian Mechanics to study life and various subjects in life sciences have been developed. In 1920s to 1950s, people realized that for organisms, the whole is greater than the parts and a purely reductionist approach seems to be inadequate and it must be combined with a holistic approach. Thus, the general systems theory (GST) was developed [9]. Von Bertalanffy's objective was to bring together under one heading the organismic science that he had observed in his work as a biologist. His desire was to use the word "system" to describe those principles which are common to systems in general. In GST, he writes: “...there exist models, principles, and laws that apply to generalized systems or their subclasses, irrespective of their kind, the nature of their component elements, and the relationships or 'forces' between them. It seems legitimate to ask for a theory, not of systems of a special kind, but of universal principles applying to systems in general" [9]. This whole process to seek the answers to fundamental questions leading to the development of modern sciences can be schematically summarized in Figure1. It is the authors' belief that by removing the philosophical contradictions between GR and QM through generalizing GST, a theory of everything (TOE) can be developed. Currently few scientists are pursuing the target [10-12].

\section{Consensus for a Scientific Theory}

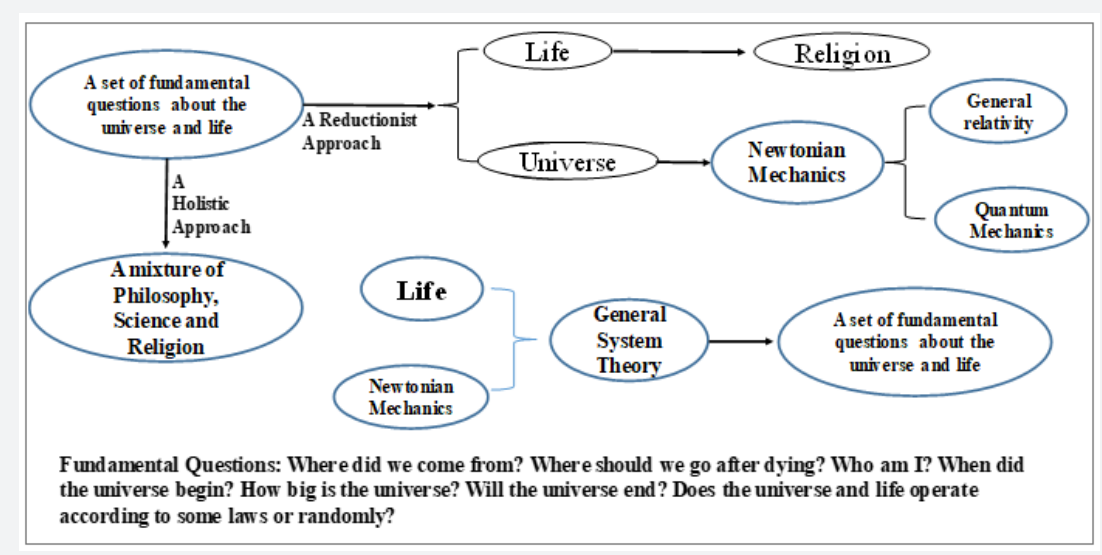

Figure 1: A Schematic Representation of the Whole Development Process of Modern Sciences. 
Researchers working on the construction of a theory of everything (TOE) can easily be criticized as a pseudoscience and therefore before we present our general ideas on the construction of the TOE, let us state what we understand as the criterion for a scientific theory. From our opinion, every scientific theory has at least three components: axioms, laws and natural phenomena. Axioms are fundamental assumptions called hypotheses. Hypotheses do not need to be proved, but if a counter example is found against one hypothesis or a paradox is derived if one accepts this hypothesis, the theory based on this hypothesis is proved to be wrong. The laws can be derived from the logical deduction from axioms or from the induction from the observed natural phenomena. Gödel [13] has proved that at least one hypothesis cannot be proved within the theoretical framework [14-16]. Thus, selection of the fundamental hypotheses is the starting point of a scientist to construct a scientific theory.

How to judge a theory to be scientific or not, people have also attempted to build some criteria. Currently, there are two criteria. One is the falsification criterion proposed by modern philosopher of science Karl Popper [17] and the other is replicability (or repeatability) as a demarcation criterion of science from pseudoscience. Braude provided a detailed discussion in an Editorial and his conclusion is that repeatability cannot be used as the criterion [18]. Cui proposed a modified criterion of falsification [19]. In different from Popper's opinion that there exist some nonfalsifiable theories, he declared that every theory is falsifiable. For example, although one may not be able to find a counter example to falsify an assumption, he may be able to derive one paradox from this assumption if this assumption is scientifically wrong. Today someone has not found the counter example does not mean tomorrow he will not be able to find. That is the nature of science. Thus, his criterion of demarcation to draw a sharp line between those theories that are scientific and those that are unscientific is dynamic. If our human beings have not falsified the hypotheses of a theory, it is still a scientific theory; otherwise if we have found a counter example or a paradox of a theory, it is unscientific. But if the application range is refined to the scope where the counter example or the paradox can be removed, it is still a scientific theory. Newtonian mechanics is a typical example and, in the future, many modern scientific theories such as GR and QM may also be subjected to this type of revision. It is our belief that science is adequate to falsify an unscientific theory but inadequate to prove it to be a truth if we define truth to be universally correct. In that sense, Popper's falsification criterion can be used to demarcate science from pseudoscience. Otherwise, it is logically inconsistent. If one can judge something to be falsified, it will have two results, one is true and the other is false. If we still retain the false within science, it conflicts with the mission of science. Of course, any individual claims to make the ultimate judgement of falsification is certainly an over-claim. He has no tool to make that judgement. As we know that scientific tool can prove the falsehood but cannot prove the axiom to be universally true. Repeat of 1000 times does not guarantee that the 1001 time will repeat again. Up to now there has not been found a counter example against the axiom, it does not guarantee there will be no counter examples against the axiom in the future. So, every axiom and law used in a scientific theory is only of relative or temporary correctness and it should not be regarded as a truth if we define the truth to be a universal law. If someone takes an axiom or a law to be a truth, it is his belief rather than the scientific evidence. The attitude itself is not very scientific since scientific spirit encourages people to question every axiom or law.

\section{General ideas on the construction of TOE}

Following Karl Popper's famous opinion, "All science is cosmology, I believe", cosmology is the foundation of all other sciences. Cosmology is intrinsically linked with mythology and religion as a quasi-rational elaboration of the former. Behind each cosmological model, it is the philosophical belief of the proposers. It is well-known that the currently most prevailing cosmological model, the Big-Bang Cosmological model (BBCM), is based on materialism and with this philosophical monism, many paradoxes can be found [20]. From the authors' point of view, the most difficult problem for a materialist belief should be the "creator problem" [21]. How was the first matter created and where did forces come from for creating this matter? This problem also exists in the modern string theory such as what are the strings in the universe and who makes them vibrate? [22] As a matter of fact, since the discovery of Einstein's famous equation, $E=\mathrm{mC}^{2}$, it has already been proved that the fundamental assumption made in materialism is wrong since matter can be transformed into energy. Schramm [23] has pointed out that very few physical theories are in such a paradoxical situation as Big-Bang cosmology which is completely based on materialism. In this monism, there are no clear definitions of matter and consciousness. In order to explain the redshift phenomenon observed, concepts such as dark matter and dark energy had to be introduced [1], but we are still unclear what they are after many decades' research $[24,25]$.

In order to overcome these paradoxes, Cui has proposed a novel cosmological model based on Buddhist philosophy (Buddhist Cosmological Model, BCM) [26] and a brief comparison was made between BCM and BBCM [27]. From his judgement, it seems that BCM is conceptually clear and logically consistent and it can explain many phenomena which belongs to the frontier problems of modern sciences. BCM has the potential that all the paradoxes encountered by BBCM can be overcome. In another paper [19], Cui discussed the issue how to apply scientific criteria to assess whether BCM is a scientific theory or a pseudoscience. The construction of TOE in this paper is basically a combination of Bertalanffy's general system theory with BCM as its philosophical foundation. 
In answering the fundamental questions posed at the beginning of this paper, many concepts should be defined and in the cosmological model based on Buddhist philosophy (BCM), it is assumed that the universe is made of energy and mind, energy is the essence of matter and of continuous nature while mind is the essence of a life and of discrete nature [26]. The universe always exists following the conservation laws of energy [28] and minds (Energy Conservation Axiom and Minds Conservation Axiom). Minds are the source of all forces in the universe since minds are vibrating all the time. The reason we interpret the life essence as mind follows the opinion of René Descartes. The discourse on consciousness has been hugely influenced by René Descartes, the French philosopher who in the mid- $17^{\text {th }}$ century declared that body and mind are made of different stuff entirely. It must be so, Descartes concluded, because the body exists in both time and space, whereas the mind has no spatial dimension [29].

The vibration of minds is responsible for all the changes and movements observed by our human beings. Each mind has the capability to accumulate the energy into matter and to decompose matter into energy. Matter is defined to have a measurable volume and mass. Mass could be static (under zero speed) or dynamic (under a non-zero speed). With this definition, the boundary between matter and energy is dynamic, as our measurement system improves, more small particles will enter the matter side. Matter can also be visible or invisible (dark to our human beings, thus we call it dark matter) and this boundary between matter and dark matter is also dynamic depending our detection ability. A life is defined as a mind with or without a body. Body could be matter or dark matter. Mind can generate, process, store and transform information and can use information to describe and communicate something with other minds. Thus, information can be thought of as a by-product of minds and can be stored in minds. Of course, information can also be stored in some media made of matter. Information can also be viewed as the resolution of uncertainty when we use it to describe something. Information can be represented as data which assigns some values to parameters or knowledge which signifies our understanding of an abstract or concrete concept. For communication, information is expressed either as the content of a message or through direct or indirect observation. For transmission, information can be encoded into various forms such as a sequence of signs or a signal. It can also be encrypted for safe storage and communication. The bit is a typical unit of information, and qubit is used in quantum information field.

For our human beings it is accustomed to use a time-space framework to describe all the phenomena we can observe and in BCM $[19,26,27,30,31,32]$ we simply adopt the definition given by Kant: "Space and time are the framework within which the mind is constrained to construct its experience of reality" [33]. It is specifically defined that the universe is the largest system our human beings can imagine, and it is assumed to be of infinite nature both in space and time (Infinite Universe Axiom). The universe can be divided into infinite number of worlds and each world is of finite nature both in time and space (Finite World Axiom) and their relationship is expressed by the following equation:

$$
\text { Universe }=\sum_{i=1}^{\infty} \text { World }_{i}
$$

Each entity in the universe from the tiniest subatomic particles to galactic superclusters and including mind-only lives, mindwith-a-body lives is cyclically operated according to the process of formation, the steady state, deterioration and decommission (Cyclic World law). This law is a direct consequence of finite world axiom. In BCM, the Big Bang is the origin of the world our human beings are living but not the whole universe. Using this definition, the awkward question of the origin of the universe can be avoided. Both the universe and worlds can be regarded as systems shown in Figure 2 and this definition emphasizes that only the universe is unknown to human beings due to its infinite nature and the system we can observe is a world which is of finite nature both in time and space and thus this system can be known by our human beings. This assumption resolves the conflict between agnosticism and knowability and may be able to combine the main points presented in the infinite universe model [34], cyclically universe model [35] and the many-worlds interpretation model [36].

$$
\text { or knowledge which signifies our understanding of an abstract or }
$$
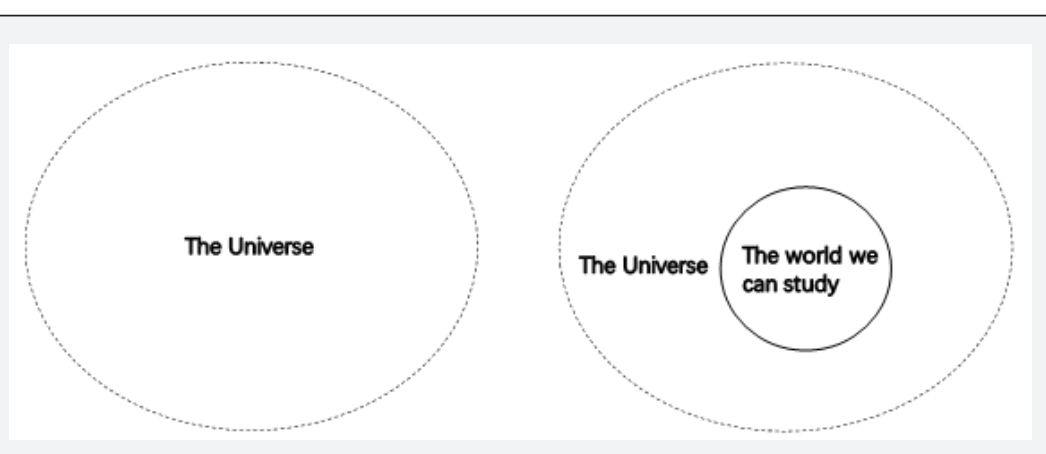

Figure 2: A Schematic Diagram for the Universe (Left) and the World We Can Study (Right).

It is assumed that everything in the world including matter and life is operated according to the Causal-Effect law (Causal-
Effect Axiom). This law is fundamentally deterministic but practically may be revealed probabilistically due to the existence 
of hidden variables. This axiom resolves the conflict between determinism and indeterminism and attributes the uncertainty to hidden variables as Bohm did [37] no matter these hidden variables are of local or non-local nature. It is assumed that the ability of a human being (more generally a life with a mind-only type or with a mind-body type) to know the external world is limited due to its finite life span (Limited ability Axiom). This axiom is an inductive law based on observations that most of human beings could not remember the knowledge learned from previous life memories, but it does not mean that human beings could not know the knowledge learned in previous lives. Currently already several means have been found to be able to recover some of the knowledge learned in a previous life such as mediumship, hypnosis, meditation and even there are some children who can be spontaneously recalling past life memories [38]. The ability of a mind to access the information stored in the universe minds is called enlightenment. Even for an enlightened person to a highest degree such as Buddha is still unable to know everything in the universe, but he can know everything for a world. The limited ability axiom is the key assumption of BCM and is more or the less the same as Gödel's law [13] that at least one hypothesis cannot be proved within the theoretical framework [14-16]. If someone could provide a counter example or derive a paradox by accepting this assumption, BCM is falsified. From the Finite World Axiom, one can immediately derive the following two laws.

a) Boundedness Law: All the systems we can study is of finite space. This is called boundedness law by Das [39] and this law is a direct consequence of the Finite World Axiom.

b) Finite Time Law: All the systems we can study is of finite time. This is called finite time law by Das [39] and this law is also a direct consequence of the Finite World Axiom.

These two laws have been pointed out in Das [39] but with a slightly different explanation. He was correctly pointed out the fact that most of the mathematical theories that we use in quantum mechanics (QM) like Fourier Transform, Laplace Transform, Linear ordinary and partial differential equations like Schrodinger's equation, linear operator theory, Hilbert space, inner products, all violate these two laws and therefore we need to investigate the consequences of this practice. Ideally all the mathematical tools we use should follow these two laws.

Let us take Laplace Transform as an example. The standard Laplace transform is defined by the following expression:

$$
L[f]=F(s)=\int_{0}^{\infty} e^{-s t} f(t) d t
$$

Since the upper limit is infinity, this definition is referred to as the Infinite Laplace Transform (ILT). Because of this infinite limit, the integral in Eq. (2) requires a convergence or boundedness condition that $\mathrm{f}(\mathrm{t})$ is restricted by the following constraint:

$$
|f(t)| \leq M e^{\alpha t}, \quad 0<M<\infty
$$

The Finite Laplace Transform (FLT) is defined as

$$
L_{T}[f]=F_{T}(s)=\int_{0}^{T} e^{-s t} f(t) d t, \quad 0<T<\infty, 0 \leq t \leq T
$$

Since the upper limit is finite, the integral in Eq. (4) always exists if $f(t)$ is a continuous function. Thus, the region of convergence of FLT is the entire complex plane. This is a significant difference of FLT to ILT of Eq. (2).

Let us assume

$$
\mathrm{f}(\mathrm{t})= \begin{cases}1 & 0 \leq t \leq T \\ 0 & \text { otherwise }\end{cases}
$$

Using the definition, Eq. (4), we can get

$$
L_{T}(1)=F_{T}(s)=\int_{0}^{T} e^{-s t} \cdot 1 \cdot d t=\frac{1-e^{-s t}}{s}
$$

We can see that the FLT has the standard ILT term 1/s, but it also has another expression involving $\mathrm{e}^{-\mathrm{sT}}$. Furthermore, in ILT, $s=0$ is a pole but in FLT, $s=0$ is not a pole but with a finite number of T. This is a very distinguishing feature of FLT. Through this comparison, it is found that both the boundedness condition, Eq. (3) and the pole problem at $s=0$ are all caused by the improper assumption of infinity. For real engineering problems, if $\mathrm{f}(\mathrm{t})$ is a continuous function within $[0, \mathrm{~T}]$, it will always be able to perform the Laplace transform and no pole will occur. Of course, more study on the consequences of using ILT is needed which is not the main topic of this paper. From the Infinite Universe Axiom and Limited Ability Axiom, we can derive the following law (or principle).

\section{General uncertainty principle (GUP)}

If a human being (or a mind) wants to know something, he must attribute all the uncertainty to the complement of that thing. For example, if we want to know the finite system (a world), we must attribute all the uncertainty to the infinite system (the universe). Other pairs of concepts are matter/energy, energy/ mind, mind/meditation, meditation/universe. So ultimately, the universe is assumed to be unknown and it exists irreducible uncertainty while the uncertainties for a given world can always be reducible. This principle could resolve the philosophical conflict between GR and QM. In different from Copenhagen interpretation of quantum mechanics who selects agnosticism for micro worlds and knowability for macro worlds, we select agnosticism for the universe and knowability for any worlds including micro worlds.

From our assessment, every existing theory seems to follow this general uncertainty principle. For example, in Buddhism, Buddha is assumed to be an enlightened person who can answer any concrete questions about a world, but he refuses to answer any questions regard the universe. These questions are called unanswered questions. In Buddhism, unanswered questions are a set of common philosophical questions that Buddha refused 
to answer. In BCM, all these questions are attributed to the characteristics of the universe. In Christianity, God is assumed to know everything, but one does not allow to question how God knows. In the famous debate between Einstein and Bohr, Einstein said, "God does not play dice" and Bohr replied "Einstein, stop telling God what to do". It is well-known that modern physical sciences are based on materialism which is a form of philosophical monism that holds that matter is the fundamental substance in nature, and that all things, including mental states and consciousness, are results of material interactions. In this theory, questions such as how the fundamental substance is originated, where the forces come from which produces all the things and makes them move and change have not been answered. Of course, GUP should be subjected to strict tests of the violations for any existing theory.

In the circulation from universe to world, from world to matter, from matter to energy, from energy to mind, from mind to meditation, through meditation one can reach his highest state to know the world, he is still unable to know everything about the universe. Therefore, GUP cannot be violated in BCM. In different from only one learning method used in modern sciences, it is pointed out that there are two ways to know the world, one is based on the data collection through measurements and the other is the enlightenment through meditation. Even one reaches the highest level of meditation, he is still unable to know everything about the universe because of its infinite nature. Thus, we only give up the right to know everything about the universe, but we gain the right to know everything about a world. It is obvious that the general uncertainty principle is a consequence of the limited ability axiom and the infinite universe axiom. It can also be regarded as the combination and generalization of Heisenberg's uncertainty principle and Bohr's complementarity principle.

From this general uncertainty principle, we can explain all the phenomena we have observed. For example, if we want to explain the phenomena related to matter, we can pass all the unknowns to energy. If we want to explain the phenomena related to energy, we can pass all the unknowns to mind. If we want to explain the phenomena related to mind, we can pass all the unknowns to meditation or enlightenment. Even one has reached the highest state of enlightenment, he can still pass the unknowns to the universe. Thus, it is concluded that we can know the world if we accept the above definitions for the universe and the world. From this GUT, we realized that knowledge is a relative concept and any measurement value is fundamentally a conditional probability.

The consciousness issue is one of the most challenging issues listed by science [29]. In fact, the philosopher David Chalmers uses the notion of mechanism to help characterize what he terms the "hard problem of consciousness." According to Chalmers [40], the easy problems of consciousness are those that can be explained in terms of computational or neuronal mechanisms. The hard problem, on the other hand, is the problem of experience itself. Most philosophers of mind use a phrase suggested by Nagel [41] to characterize this purely subjective aspect: there is something it is like to be a conscious organism. The phrase "there is something it is like to be a conscious organism" obviously doesn't identify anything we can objectively test. And that is indeed the chief problem of consciousness: Its inherently subjective nature makes it very difficult (perhaps impossible) to fit it into our objective understanding of the world. Chalmers, Nagel, and others go so far as to argue that a purely physicalist or materialist framework cannot account for consciousness. They have argued that consciousness most likely is fundamental in some sense, not emergent from matter. They see this move as necessary because they see consciousness as essentially anomalous with respect to our conventional, physicalist understanding.

Since we do not know much about the consciousness after many decades research, the opinions presented in Buddhist philosophy are worth to be taken as a reference. American physicist and parapsychologist Targ commented "I think it is interesting to consider some of the ideas from Buddhism, because of their great density in Buddhist writing, and their close agreement with much that we see in the laboratory" [42]. According to Buddhism [3], consciousnesses are functions of a life to feel the external world and internal body. Any lives with a mind-only type or with a mind-body type including plants, animals, human beings and even extraterrestrial intelligence have consciousnesses. Human's consciousnesses can be divided into 8 types, they are consciousnesses at eyes, ears, nose, tongue, body and brain, the manas consciousness, and finally the alaya consciousness. Only the alaya consciousness will continue to exist after dying and it is the source of life forces and it stores all the karmas in the previous life history. That is why this alaya consciousness is defined as mind in BCM which is of energy nature while the former seven consciousnesses are related to the body. When one is dying, the consciousnesses at eyes, ears, nose, tongue, body and brain will be lost. The function of the seventh consciousness is the bridge between the former six consciousnesses with the mind. The dying process is a process of the separation of the mind from the body. So, mind is the energy while other seven consciousnesses are related to the body. In BCM, both plants and animals also have minds and some types of consciousnesses but may not have as many as human beings. Thus, in order to explain all the phenomena related to consciousnesses, we can pass the unknowns to mind. Since mind cannot be created and destroyed by human beings, so any robot which does not have a mind will not be regarded as a life no matter how intelligent the implemented software is. However, human beings can have the ability to create a body through other technical means such as cloning and a mind may enter that body, through this way, human beings can produce lives in addition to the traditional birth method. These lives should have the equal right as human beings. 
There are three types of life in the world we are living, the mind only, the mind with an invisible body (also dark matter to our human being) and the mind with a visible body (being plants, animals and human beings) and these three types of lives can also be divided into six categories according to their happiness, Heaven, Asura, Human being, Animal, Ghost, Hell [43]. Only plants, animals and human beings are with a sensible body by our human beings. Non-existence of other types of lives is also a belief or an over-claim rather than a scientific proof. Currently, there are several means to prove the existence of other types of lives. For example, mediumship can prove the existence of ghosts [38] while Si-Chen Lee has developed a special method to communicate with extraterrestrial intelligence through finger reading and psychokinesis [44]. According to Buddhism [3], lives with only mind exists in the Heaven, they are the highest level of life in the world we are living while the lives in the Hell is the lowest level in the world we are living. So according to this BCM, human beings are not the most intelligent creatures in the world we are living but in the middle level. All the matter used by other four types of lives and together with their bodies belong to dark matter.

For any lifeless object from a particle to a star in the world we are living, it is created by minds. No matter whether we can see (visible matter) or not (dark matter), it will experience the cycle of formation, the steady state, deterioration and decommission and for each individual life, no matter whether we can see (human beings, animals and plants) or not (other four types of lives), he will be reincarnated within the six types of lives in the universe. Here it must be pointed out that according to Buddhism, reincarnation of a life is not confined to the world he is living, but in the whole universe. Even for the mind only life in the Heaven, he will also have a life span and can be reincarnated into other five types with a body in the universe, not necessarily in the same world as he lived in a previous life. Reincarnation of all lives is a law of nature
[44]. So, in this BCM, parents provide only the bodies to their children and not the life. We are very soon reached a stage that we can clone our bodies, but the essence of life does not change, and any lives produced this way should have the same rights as us.

The dual model of a human being with body and mind are adequate to explain all the anomalous phenomena reported in the book [38] such as near-death experience (NDE), out of body experience (OBE), mediumship and children claiming past-life memories etc. As discussed above, in BCM, information is generated by minds and used by minds for communication. Information stored in minds cannot be destroyed while information stored in a material media can be destroyed. Information can be transmitted through energy, matter and entanglement of minds. It is a wellknown fact that information could also exert influence on the behavior of a life. Thus, for an open system it must consider the exchange of energy, mind, matter, dark matter and information at the system boundary shown in Figure 3. Energy and mind are fundamental elements while matter, dark matter and information are products made by minds. In BCM, it is emphasized that there never exists any closed or isolated system in a world [31]. Since the universe is infinite, it is meaningless to discuss any nature of the universe, i.e., whether the universe is a closed system or an isolated system or an open system. Since all the systems our human beings can study should be in a world, no matter how big it is, such as the Milky Way or even larger Galaxy system, it is an open system in nature since we could not prevent the exchange of information, mind, energy and even dark matter at the system boundary. Therefore, the assumption of a closed system or an isolated system is purely mathematical and does not represent the reality. Thus, all the laws such as the three laws of thermodynamics derived by employing a closed system assumption should be subjected to the scrutinization to identify their application ranges.

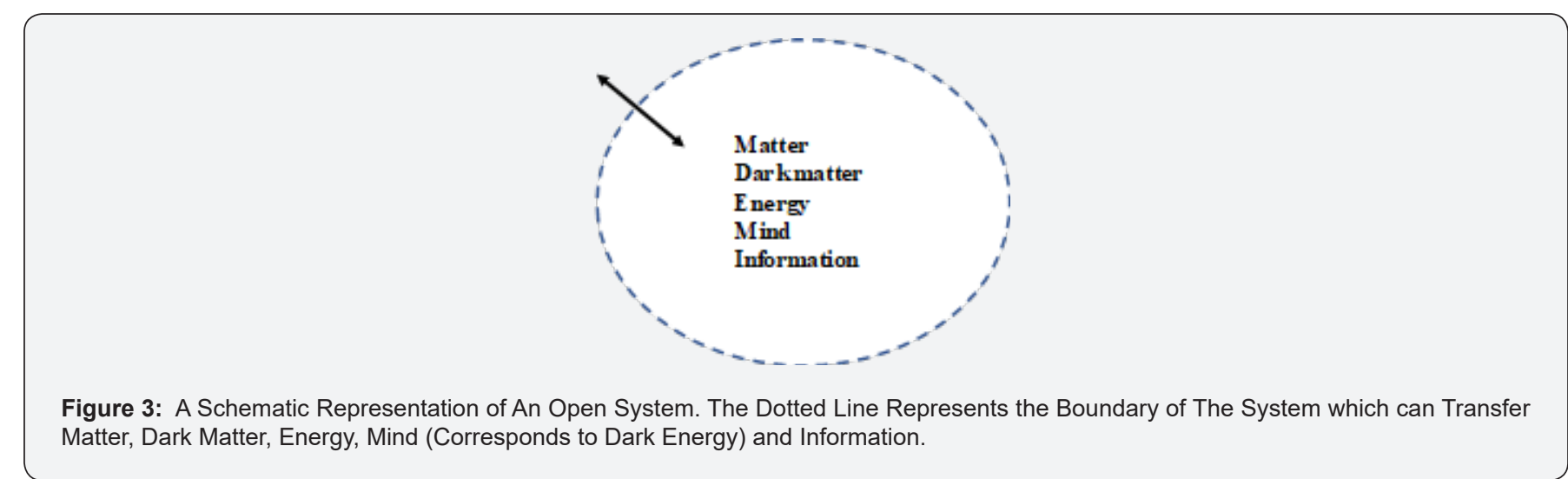

Based on above 6 axioms (Energy conservation axiom, minds conservation axiom, infinite universe axiom, finite world axiom, causal-effect axiom, limited ability axiom), a theory of everything (TOE) can be constructed by treating the object to be studied as an open system. Then all the approaches used in GST can also be used in TOE. This TOE can explain all the phenomena observed in a world and predict the future phenomena for the world. A system is a group of interacting or interrelated entities that form 
a unified whole. A system is delineated by its spatial and temporal boundaries, surrounded and influenced by its environment, described by its structure and purpose and expressed in its functioning. Systems are the subjects of study of systems theory. Figure 4 is a schematic diagram of a general system. Since every problem to be studied can be treated within a system framework and therefore, the general system theory (GST) proposed by Bertalanffy [9] can be regarded as a universal method of revealing how the world operates [46]. Regarding any object to be studied as a system, any system includes five elements, namely, the system itself, constraint conditions, boundary support conditions, input and output. Given the system, constraints and boundary support conditions, the output will be a function of the input, and the function $\mathrm{f}$ is called the transfer function. This function can always be revealed by us humans either accurately or approximately. The difference between Bertalanffy's GST and present TOE is their philosophical foundation. GST is based on materialism while TOE is based on Buddhism.

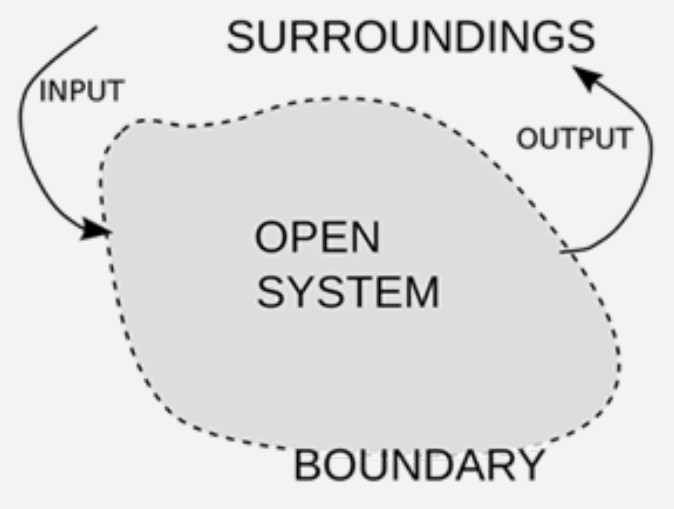

Figure 4: A Schematic Diagram of a General System.

\section{Special Issues Resolved in TOE}

In this section, some typical problems are solved in this TOE as a demonstration.

\section{Wave-particle duality}

Wave-particle duality is the concept in quantum mechanics that every particle or quantum entity may be described as either a particle or a wave. It expresses the inability of the classical concepts "particle" or "wave" to fully describe the behaviour of quantum-scale objects. Through the work of Max Planck, Albert Einstein, Louis de Broglie, Arthur Compton, Niels Bohr, and many others, current scientific theory holds that all particles exhibit a wave nature and vice versa. This phenomenon has been verified not only for elementary particles, but also for compound particles like atoms and even molecules. For macroscopic particles, because of their extremely short wavelengths, wave properties usually cannot be detected. Although the use of the wave-particle duality has worked well in physics, the meaning or interpretation has not been satisfactorily resolved.

In TOE based on BCM, a small particle is interpreted as an unstable structure. Since every particle is created by mind or minds by accumulating the energy and if the particle is still in an unstable structure state, it needs the mind or minds to hold them together. Since mind is vibrating all the time, thus, the particle is changing between holding and unholding. The holding state is a particle state while the unholding state is an energy state which shows the wave characteristics. If the structure is stable which means that even if the mind is left the structure, the structure is still there, and it becomes independent of the mind. If the structure is strong enough, the forces from a mind or several minds have negligible influence on the structure, the wave property will disappear.

\section{Quantum entanglement}

Quantum entanglement is a label for the observed physical phenomenon that occurs when a pair or group of particles is generated, interact, or share spatial proximity in a way such that the quantum state of each particle of the pair or group cannot be described independently of the state of the others, even when the particles are separated by a large distance. The topic of quantum entanglement is at the heart of the disparity between classical and quantum physics.

The problem of quantum entanglement is one of the most intriguing problems of the current quantum theory and it is believed that its solution will give us a new insight into the nature of the quantum world [47] and is a key resource for quantum information processing (QIP) [48]. In order to explain this phenomenon, a wave function collapse concept is introduced which is the most difficult part of quantum mechanics theory to be understood and it needs to make a claim that quantum behaviour is significantly different from macroscopic object behavior. In 
TOE based on BCM, this is just attributed to the fundamental properties of minds and it is not the entanglement of particles but the entanglement of minds. Since with unstable particles the minds are still with the structure and they can be entangled. Figure 5 is a schematic illustration for the entanglement of two unstable structures and it does not depend on the scale of the structure such as micro-structure or macro-structure. Instead, it depends on the stability of the structure or more specifically speaking whether there is a mind with the structure. A general trend may be observed, the smaller the structure, the less stable of the structure, but this is only an empirical observed phenomenon but not a strict rule. The speed to transform information from a mind to another mind could be superluminal and any behavior related to minds must be studied in other methods such as meditation.

\section{Entangl ement between mind $A$ and mind B \\ structure is \\ holding \\ together by \\ mind $\mathrm{A}$. \\ structure is \\ holding \\ together by \\ mind $B$.}

Figure 5: A Schematic Illustration of an Entangled System.

\section{Measurement problem}

The measurement problem in quantum mechanics is the problem of how (or whether) wave function collapse occurs. The inability to observe such a collapse directly has given rise to different interpretations of quantum mechanics and poses a key set of questions that each interpretation must answer.

In BCM, it is held that any mathematics is an idealized or abstract representation of a reality. This reality itself has no mathematics such as wave functions or even numbers. In order to measure the value for a particular property, such as the height of a person, we need the apparatus and the persons who use the apparatus to do the measurement. Before the measurement, quite a lot of values are possible and a probability distribution or possibility distribution functions can be used to describe the value of the parameter but after the measurement, a much narrower gap and in the limit a deterministic value may be assigned to this parameter. Both the measurement accuracy of the apparatus, the measurement method and the person's carefulness and skill are possible factors to affect the measurement results and for micro particles, these influences could be more significant and it is very hard to repeat the tests with the same measurement value. That is the essence of the measurement problem and wave function collapse is just one of the possible explanations.

\section{Psi phenomena}

Whether human beings have the so-called parapsychological (psi) phenomena or not is directly related to the functions of the mind. From the founding in 1882 of the Society for Psychical Research, research on psi has used or even developed scientific practices, with the aim to "examine without prejudice or prepossession" the nature of these phenomena. The study of purported psi phenomena using the scientific method is defined as a new subject area called Parapsychology. The Parapsychological Association, the professional association of the field, has been an affiliate of the American Association for the Advancement of Sciences (the world's largest general scientific society) since 1969.

Psi typically includes two major areas:

a) what used to be called extrasensory perception (ESP)

b) psychokinesis (PK)

ESP includes purported telepathy (being affected by someone's thoughts or emotions, unmediated by the senses or logical inference, such as guessing more accurately than would be expected by chance who sends you an e-mail unexpectedly), clairvoyance (obtaining information about a distant state of affairs, unmediated by the senses or logical inference, such as in remote viewing (RV) in which someone accurately describes details of a place chosen at random by someone else), precognition/presentiment (being affected by an event taking a place in the future that could not have been foreseen, as in dreaming about planes crashing against tall buildings the night before 9/11), and retrocognition (having noninferable knowledge about a past event). PK refers to putative direct action of mental events (e.g., intention) on physical objects, unmediated by muscular or indirect mechanical activity. There is macropsychokinesis (or anomalous force), an effect on observable objects such as a table levitating without any apparent mechanical explanation, and micropsychokinesis (or anomalous perturbation), an effect on small, unobservable events, such as mentally affecting the output of a random number generator that otherwise produces random outputs. Some psi researchers 
study the possibility of consciousness surviving death, including studies of children who spontaneously report information about a past life to which neither they nor those close to them apparently had access. Both descriptive and experimental approaches can be employed to evaluate psi phenomena. Cardeña carried out a comprehensive integration of current experimental evidence and theories about psi phenomena and concluded that the evidence provided cumulative support for the reality of psi, which cannot be readily explained away by the quality of the studies, fraud, selective reporting, experimental or analytical incompetence, or other frequent criticisms [49]. The evidence for psi is comparable to that for established phenomena in psychology and other disciplines, although there is no consensual understanding of them. The psi capability is a support to our assumption that mind has the capability to accumulate energy into matter and to decompose matter into energy and is the source of all forces in the universe. Furthermore, a human being could have more psi abilities such as communicating with alien intelligence reported in Lee [14].

\section{Utilization of dark matter and dark energy}

One of the most challenging problems in modern physics and cosmology is the dark matter and dark energy. By assuming the current cosmological model is correct, the universe contains $68 \%$ dark energy, 27\% dark matter and conventional energy and matter only occupies $5 \%$. However, after many decades' search, we have not found any clues what is the dark matter and what is the dark energy. In TOE based on BCM, we gave a different interpretation to dark matter and dark energy.

Dark energy is simply interpreted as the universal minds and they are the sources of forces which can do work. Minds can accumulate energy into matter and can make matter objects move and change. Minds can generate, process, store and transform information. A life can be a mind with a body or a mind only. If a life with a body, then its movement is greatly restricted in the universe while a mind without a body can be anywhere in the universe. Any life can store and transform information and thus, one mind is asking for the help from other minds especially those mind-only or mind-with-a dark-body lives can be interpreted as the utilization of dark energy while a human being is trained to consume food in dark matter can be interpreted as the utilization of dark matter. There are reports that some persons can survive many years without eating any food or water. These persons can be regarded as the utilization of dark matter.

\section{Summary and Conclusion}

Currently few persons are working on the development of a theory of everything [10-12] and now, these different theories of everything are very different. If a theory of everything is possible, it is still in the infant stage. In this paper, another novel version of the theory of everything is proposed and the main idea is to change the philosophical foundation of the general system theory from materialism to Buddhism. The main points of our TOE can be summarized as follows:

The key differences between materialism and Buddhism is that the essence of the universe is energy and mind (dualism) rather than fundamental particles (monism). Particles are formed by a mind (or several minds) to accumulate energy to a measurable degree and a mind (or several minds together) can also decompose a particle into energy. The essence of a lifeless object is energy while the essence of a life is mind. It could have three types of lives in the universe, mind-only, mind with a dark body and mind with a body. TOE based on BCM assumes that every scientist is subjected to the limit ability axiom and the universe is of infinite nature both in time and space (infinite universe axiom). From these two axioms, we have derived a general uncertainty principle which can resolve all the philosophical conflicts between infinite/ finite, determinism/ indeterminism, agnosticism/ knowability.

Using the general uncertainty principle, we can explain all the phenomena we have observed. For example, if we want to explain the phenomena related to matter, we can pass all the unknowns to energy. If we want to explain the phenomena related to energy, we can pass all the unknowns to mind. If we want to explain the phenomena related to mind, we can pass all the unknowns to meditation or enlightenment. Even one has reached the highest state of enlightenment, he can still pass the unknowns to the universe. Thus, it is concluded that we can know the world if we accept the above definitions for the universe and the world. From this GUT, we realized that knowledge is a relative concept and any measurement value is fundamentally a conditional probability. In TOE, every system we can study is of open nature and it could have exchanges of five types of quantities, energy, mind, matter, dark matter and information. Within the five quantities, energy and mind are the two fundamental elements while the other three are the products made by minds. Obviously, the present percentage such as $68 \%$ dark energy and $27 \%$ dark matter are derived using Big-Bang cosmological model and it needs recalculation using TOE.

In TOE, information can be transformed through three means, matter, energy and entanglement of minds and the third method can be superluminal and this will be the future development. Mind can also have the potential to utilize dark energy and dark matter and this is also an area of future importance. In order to understand the behavior of minds, one must rely on the method of meditation. In TOE, all the fundamental questions can be addressed and based on causal-effect law and the reincarnation of life, a perfect life model was proposed [32].

\section{Acknowledgement}

This work was supported by the General Program of National Natural Science of China "A study on the water absorption 
property of the buoyancy material for the full ocean depth manned submersible" (Grant No. 51879157), the "Construction of a Leading Innovation Team" project by the Hangzhou Municipal government, the Startup funding of New-joined PI of Westlake University with grant number (041030150118).

\section{References}

1. Wilczek F (1982) Old and new relics in cosmology (A Review). Proc. Natl Acad. Sci. USA 79(10): 3376-3379.

2. Greenstein JL (1993) An ancient revisits cosmology. Proc Natl Acad Sci USA 90(11): 4878-4881.

3. Stephen LJ (2008) An Introduction to Buddhist Philosophy. Cambridge University Press, New York, USA.

4. Cui WC, Blockley DI (1990) Interval probability theory for evidential support. Int J of Intelligent Systems 5(2): 183-190.

5. Kelvin L (1931) I Nineteenth century clouds over the dynamical theory of heat and light. Philosophical Magazine Series 2(7): 1-40.

6. Heisenberg W (1927) The physical content of quantum kinematics and mechanics. In: Physik Z, Wheeler JA, Zurek WH Quantum theory and measurement, Princeton university press 43: 172-198.

7. Heisenberg W (1930) The physical principles of the quantum theory Eckart C, Hoyt FC, Dover publications, University of Chicago.

8. Whitaker A (2006) Einstein, Bohr and the Quantum Dilemma - From Quantum Theory to Quantum Information. $2^{\text {nd }}$ Edition, Paperback, Cambridge University Press, UK.

9. Bertalanffy LV (1968) General System Theory: Foundations, Development, Applications, George Braziller, New York, USA.

10. De Aquino F (1999) Theory of Everything.

11. Steven W (2011) Dreams of a Final Theory: The Scientist's Search for the Ultimate Laws of Nature. Knopf Doubleday Publishing Group.

12. Stephen HW (2006) The Theory of Everything: The Origin and Fate of the Universe. Phoenix Books, Special Anniv.

13. Gödel K (1931) About formally undecidable sentences of the Principia Mathematica and related systems I. Monthly magazines for mathematics and physics 38: 173-198.

14. Walach H (2019) Beyond a Materialist Worldview. Towards an Expanded Science. First published by the Scientific and Medical Network.

15. Basios V, Bouratinos E (2006) Godel's other legacy and the imperative of a self-reflective science. Kurt Goedel Society Collegium Logicum 9: $1-5$.

16. Devlin K (2002) Kurt Gödel - Separating truth from proof in mathematics. Science 298(5600): 1899-1900.

17. Popper K (1963) Conjectures and Refutations: The Growth of Scientific Knowledge (2002 ed.), London: Routledge.

18. Stephen EB (2018) Editorial. Journal of Scientific Exploration 32(2): 255-264.

19. Cui WC (2019d) Some Discussions on the Establishment of a Scientific Cosmological Model. Ann Rev Resear 5(1).

20. Clark M (2007) Paradoxes from A to Z. $2^{\text {nd }}$ Edition, Taylor \& Francis e-Library.

21. Sarfati JD (1998) If God Created the Universe, Then Who Created God? CEN Tech J 12(1): 20-22.
22. Zwiebach B (2004) A First Course in String Theory. Cambridge University Press.

23. Schramm DN (1998) The age of the universe, dark matter, and structure formation. Proc Natl Acad Sci. USA 95 p. 1.

24. Neta AB (2015) Dark matter universe, Proc Natl Acad Sci USA 112(40): 12243-12245.

25. Arun K, Gudennavar SB, Sivaram C (2017) Dark matter, dark energy, and alternate models: a review. Advances in Space Research 60(1): 166-186.

26. Cui WC (2019b) On A Logically Consistent Cosmological Model Based on Buddhist Philosophy. Ann Soc Sci Manage Stud 3(1): 28-32.

27. Cui WC (2019c) A Comparison of BCM with BBCM. Ann Soc Sci Manage Stud 3(3): 66-70.

28. Das S (2012) Conservation Laws of Nature. Journal of Applied Global Research 5(12): 1-12.

29. Greg M (2005) What Is the Biological Basis of Consciousness? Science 309(5731): 1-79.

30. Cui WC (2019a) A Simple Idea on the Unification of Einstein-Bohr Controversy. Ann Soc Sci Manage Stud 2(5): 120-121.

31. Cui WC (2019e) On Conservation Laws for an Open System Based on BCM. Ann Rev Resear 5(1).

32. Cui WC (2019f) On Faith or Belief. Ann Soc Sci Manage Stud 4(3).

33. Kant I (2002) Metaphysical Foundations of Natural Science. in Theoretical Philosophy after 1781, edited by Henry Allison and Peter Heath, translated by Michael Friedman, Cambridge: Cambridge University Press.

34. Borchardt G (2017) Infinite universe theory (Version 20180525). Berkeley, CA, Progressive Science Institute.

35. Paul SJ, Turok N (2002) A Cyclic Model of the Universe. Science 296(5572): 1436-1439.

36. Bryce SD, Neill GR (eds) (1973) The Many-Worlds Interpretation of Quantum Mechanics. Princeton Series in Physics, Princeton University Press.

37. Bohm D (1952) A suggested interpretation of quantum theory in terms of hidden variables. Physical Review 85(2): 166-189.

38. Moreira AA, Santos FS (2012) Exploring Frontiers of the Mind-Brain Relationship. Springer, New York, Dordrecht Heidelberg, London.

39. Das S (2013a) Assumptions in Quantum Mechanics. International Journal of Theoretical and Mathematical Physics 3(2): 53-68.

40. Chalmers DJ (1996) The Conscious Mind: In Search of a Fundamental Theory. Oxford University Press.

41. Nagel T (1974) What Is It Like to Be a Bat? The Philosophical Review 83(4): 435-450.

42. Targ R (2019) What Do We Know about Psi? The First Decade of Remote Viewing Research and Operations at Stanford Research Institute. Journal of Scientific Exploration 33(4): 569-592.

43. Harvey P (2013) An Introduction to Buddhism. Teachings, History and Practices, $2^{\text {nd }}$ Edition, Cambridge University Press, Cambridge, New York, Melbourne, Madrid, Cape Town, Singapore, São Paulo, Delhi, Mexico City.

44. Chen LS (2018) The science of spirituality. Taibei: Suncolor (in Chinese).

45. Das S (2013b) Reincarnation- a Law of Nature. International Journal of Modern Engineering Research (IJMER) 3(2): 1192-1211. 
46. Chen D, Walter MS (1993) General System Theory: Toward a Conceptual Framework for Science and Technology Education for All. Journal of Science Education and Technology 2(3): 447-459.

47. Samuel LB, Lo HK (2001) Scalable Quantum Computers: Paving the Way to Realization. Wiley-VCH, New York.
48. Imai H, Hayashi M (2006) Quantum Computation and Information. (Eds.) From Theory to Experiment, Springer-Verlag Berlin Heidelberg.

49. Cardeña E (2018) The Experimental Evidence for Parapsychological Phenomena: A Review. American Psychologist. Advance online publication 73(5): 663-677.

\section{Your next submission with Juniper Publishers} will reach you the below assets

- Quality Editorial service

- Swift Peer Review

- Reprints availability

- E-prints Service

- Manuscript Podcast for convenient understanding

- Global attainment for your research

- Manuscript accessibility in different formats

( Pdf, E-pub, Full Text, Audio)

- Unceasing customer service

Track the below URL for one-step submission https://juniperpublishers.com/online-submission.php 